\title{
Is gravitational energy real?
}

\section{The long-recognized problem of Einstein's theory of gravitation, that gravitational energy is not unambiguously defined, may be unavoidable. But people keep trying.}

BetweEn Newton's time and the beginning of the twentieth century, most people were broadly speaking content with the notion of the potential energy of an object in a gravitational field. Following the Galileo legend, one had merely to drop a stone from a height to see its potential energy converted into kinetic energy. By the nineteenth century, the interplay between the two had become a way of solving practical problems, most elegantly by means of hamiltonian mechanics, but more crudely (as with a falling stone) by equating decreases of potential energy with concomitant increases of kinetic energy. But Einstein changed all that.

It is paradoxical that the best theory of gravitation yet has thrown gravitational potential energy into a confusing limbo. $\mathbf{R}$. Penrose, in a recent paper (Proc. $R$. Soc. A381, 53; 1982) puts the point directly, if inelegantly, by noting that "it is perhaps ironic that energy conservation, a paradigmatic physical concept arising initially from Galileo's (1638) studies of the motion of bodies under gravity... should nevertheless have found no universally applicable formulation, within Einstein's theory, incorporating the energy of gravity itself". But people keep trying to define gravitational energy. The latest attempt of this kind, by D. Lynden-Bell and J. Katz (Mon. Not. R. astr. Soc 213, 21; 1985), is intriguing chiefly as an illustration of the difficulties.

The underlying problem with gravitational potential energy in general relativity stems from the principle of equivalence, which logically enters the theory as an assumption, and which has as one of its consequences that an observer falling freely in a gravitational field will have the illusion that the field does not exist. In other words, it is always possible to get rid of a gravitational field by the choice of a suitably accelerated frame of reference. The principle of equivalence leads to the conclusion that free-falling objects always have the same acceleration (which is Galileo's observation). But if gravitational acceleration can always be transformed away, gravitational potential energy cannot have the significance attributed to it in classical mechanics.

Einstein naturally took up the question of how the classical conservation laws would be transferred into his new formalism. One of the frustrations that quickly became apparent is that this seemed not to be unambiguously possible, at least in the strict sense that the representation of a physical quantity should be independent of the coordinates in which this is done (or, technically, that its numerical value at any point should transform with changes of coordinates as does a tensor quantity).

The situation is really rather odd. What Einstein's general theory does is to relate the quantities describing the curvature of four-dimensional space (and which themselves are functions of the space and time coordinates) to a quantity called the energy-momentum tensor, a true physical quantity (in the sense of being a tensor and thus coordinate-free) which represents the energy and the momentum locked up both in matter and in fields other than the gravitational field, electromagnetism for example. Einstein's problem, in the years succeeding 1917, was to find some way of representing the total energy of a system - matter-energy, the energy of the electromagnetic field and of other fields as well as the energy of the gravitational field, together with the modern analogue of the old potential energy - so as then to show that this quantity is conserved.

Einstein recognized that his solution is not ideal. The best candidate for a conserved quantity representing energy is not strictly a tensor but, a construct from the quantities describing the curvature of space-time which is not a tensor in the strict sense but; a pseudo-tensor, viz a quantity depending on the coordinate system is Einstein's pseudo-tensor, not a quantity that exhibits on a microscopic scale the conservation of energy and of momentum.

So is there a buried scandal in general relativity? Einstein's failure (often repeated) to find a quantity representing energy and momentum whose microscopic conservation is self-evident does not imply that energy and momentum are not macroscopically conserved. The difficulty that immediately raises its head is that the verification of macroscopic energy conservation must necessarily be possible only within the framework of a particular macroscopic description of the Universe. And many model universes in which energy is not conserved, which are nevertheless inconsistent with energy conservation, have been constructed.

Are there some circumstances in which the definition of potential energy, or the energy of the gravitational field, is possible in terms of the variables that define that field? This is the path that Lynden-Bell and
Katz have followed. Beginning with an observation due to J.A. Wheeler that the problem of the unambiguous definition of potential energy may be simpler in the conditions in which spherical symmetry applies, they seek to define the gravitational field energy unambiguously in such circumstances.

The argument goes like this. In a system in which there are only gravitating masses, there must be some quantity, a function of the space-time coordinates, whose integral over any space-like surface (say that corresponding to the present time) is equal to all the energy associated with the mass, its rest-energy and momentum, and its gravitational potential energy. The equations of general relativity allow calculation of the rest-energy from the descriptors of the curvature, so that the difference between the surface integral and the mass within the surface must be the gravitational potential energy. Thus, by considering the difference between the surface integral on two nearby space-like surfaces, it should be possible to define the density of gravitational potential energy, the field energy. Lynden-Bell and Katz pin down their calculation by making an artificial cut in space-time along some space-like surface in which, without loss of generality, they can suppose that spacetime is flat.

Physically, the result makes sense. The energy locked up in the gravitational field (as distinct from the mass itself) does indeed decrease with distance from massive objects. Significantly, the field around a black hole turns out to contain the whole energy of the condensed object. This fits in well with the views of those who hold that the significant consequences of black holes are external. Lynden-Bell and Katz say that their result differs from that of the "Penrose school", a reference to Penrose's elegant generalization of general relativity to accommodate particles with spin. That, according to Lynden-Bell and Katz, puts all the energy of a black hole inside it.

The implications of this calculation will depend on whether it can be generalized to universes that are neither spherically symmetrical nor static. There seems to be good reason for hoping that something can be done. But if part of the essence of general relativity is that potential energy cannot be unambiguously defined except by reference to large-scale structure, there is bound always to be a degree of arbitrariness in the way in which the concept arises.
John Maddox 